\title{
CFD SIMULATION OF THERMO-AERAULIC FIELDS IN A CHANNEL WITH MULTIPLE BAFFLE PLATES
}

\author{
Y. Menni ${ }^{1, *}$, A. Azzi ${ }^{2}$, C. Zidani ${ }^{1}$
}

\begin{abstract}
In this paper, a two-dimensional incompressible flow of a Newtonian fluid through a horizontal duct of rectangular section, where four flat rectangular baffle plates were inserted and fixed to the top and bottom walls in a periodically staggered manner, is examined and analyzed numerically using the finite volume method by means of commercial CFD software FLUENT 6.3. Researchers consider this situation as a significant issue in the field of heat exchangers, for which the fluid flow characterization, heat transfer and skin friction loss distribution, along with the existence and the extension of possible re-circulations must be determined. The aspect ratio of channel width-to-height, channel length-to-hydraulic diameter, baffle spacing-to-channel height ratio, and blockage ratio of baffle height-to-channel height are fixed at $\mathrm{W} / \mathrm{H}=1.321, \mathrm{~L} / \mathrm{D}_{\mathrm{h}}=5.137, \mathrm{Pi} / \mathrm{H}=0.972$, and $\mathrm{h} / \mathrm{H}=0.547$, respectively. The Reynolds-Averaged Navier-Stokes Equations are the governing flow equations for the problem investigated, with the energy equation. In particular, flow and temperature fields, dimensionless axial velocity profiles, skin friction coefficients, local and average Nusselt numbers, and thermal enhancement factor were presented at constant wall temperature condition along the upper and lower channel walls. The presence of the baffle plates in the whole domain analyzed causes a much high skin-friction loss, $\mathrm{f} / \mathrm{f}_{0}=10.829-25.412$ but also provides a considerable heat transfer increase in the duct, $\mathrm{Nu} / \mathrm{Nu}_{0}=3.623-5.008$, depending on the Re values. The enhancement thermal factor for fluid flowing in the baffled channel with larger flow rate is found to be higher than that with smaller flow rate. The enhancement thermal factor augments with the rise of Reynolds number and thus, the highest Reynolds number value, $\mathrm{Re}=32,000$, provides maximum thermal performance factor, $\mathrm{TEF}=1.783$. This indicates that the introducing the flat rectangular baffle plates into the flow in a staggered arrangement can improve the heat transfer efficiency inside the channel.
\end{abstract}

Keywords: Computational Fluid Dynamics, Heat Transfer, Friction Loss, Thermal Enhancement Factor, Shell-and-Tube Heat Exchangers, Renewable Energy, Solar Air Collectors

\section{INTRODUCTION}

In general, in order to achieve high energy performances, it is essential to install rows of baffles and fins within the flow channel in the heat exchangers so as to create turbulence and also to lengthen the trajectory of fluids by promoting a better convective heat exchange. Consequently, a remarkable improvement in the thermal efficiency is obtained. In literature, numerous investigations on baffled channel heat transfer are reported, but only the relevant papers are cited here.

The first study on the numerical analysis of the features of the flow and forced-convection heat transfer in periodically varying cross section ducts was reported by Patankar et al. [1]. The authors exposed the concepts of periodically fully-developed flow and heat transfer.

The three-dimensional laminar forced-convection flow was investigated by Guo and Anand [2] in a duct having one single baffle in the intake region. The impact of the Reynolds number, Prandtl number, baffle height and thermal conductivity ratio were examined. In general, both separation and recirculation lengths around baffles increase with an increase in the flow Reynolds number and baffle height. In addition, the spanwise averaged Nusselt number increased with an increase in the thermal conductivity of the wall.

A numerical and experimental study was carried out by Demartini et al. [3] on air flow inside a channel with a rectangular section with two baffle plates mounted on the upper and lower walls. This study included also 
a comprehensive investigation of the velocity and pressure profiles. The solution to the problem was found by the Hot Wire Anemometry technique and the finite volume method using the CFD program FLUENT 5.2.

Gajusingh et al. [4] experimentally investigated the impact of a baffle, which acts as a vortex generator, on the heat transfer in a smooth circular tube, using the Particle Image Velocimetry (PIV) technique. Results showed that the turbulent velocities were enhanced by a factor of two to three and the rates of energy production and dissipation were enhanced by more than an order of magnitude when a baffle was inserted in the channel. The most significant turbulence enhancement was observed in the region within a distance of two baffle heights from the bottom wall just downstream of the baffle which increased to three baffle heights at further downstream locations.

Nasiruddin and Kamran Siddiqui [5] indicated that the convective heat transfer in a heat exchanger tube may be enhanced by placing a baffle inside the tube. The investigators considered a comparative study between three different baffle orientations. The first case examined a vertical baffle. The second case investigated a baffle inclined towards the upstream end, and the third one considered a baffle inclined towards the downstream end. The results suggested that a baffle inclined towards the downstream side with a smaller inclination angle $\left(15^{\circ}\right.$ in their study) is a better choice as it enhances the heat transfer by a similar magnitude with a minimal pressure loss.

Karwa and Maheshwari [6] investigated fully (open area ratio of $46.8 \%$ ) and half (open area ratio of $26 \%$ ) perforated baffles covering $R e$ values ranging from 2,700 to 11,150. The study showed an enhancement of 79$169 \%$ in Nusselt number over the smooth duct for the fully perforated baffles and $133-274 \%$ for the half perforated baffles while the friction factor for the fully perforated baffles are 2.98-8.02 times of that for the smooth duct and is 4.42-17.5 times for the half perforated baffles. In general, the half perforated baffles are thermo-hydraulically better to the fully perforated baffles at the same pitch. Of all the configurations studied, the half perforated baffles at a relative roughness pitch of 7.2 give the greatest performance advantage of $51.6-75 \%$ over a smooth duct at equal pumping power.

Another way for improving the heat transfer characteristics in thermal systems is the use of porous medium [7]. This technique has received a considerable attention and has been the subject of many investigations. Among them, Santos and de Lemos [8] performed simulations in order to investigate and document a numerical analysis on laminar flow and heat transfer along a channel with porous and solid baffles. The numerical results for the friction factor and for the Nusselt number were compared with available data, indicating that results herein differ by less than $5 \%$ in relation to published results. Further simulations comparing the effectiveness of the porous material used showed that no advantages are obtained by using low-porosity and low-permeability baffles in the laminar flow regime.

The most important parameter for improving the heat transfer performance is the shape of the obstacles. The obstacle geometry reconfiguration can be produced by casting, machining, forming, welding, and other methods [9]. Various obstacle shapes and their orientations have been reviewed and investigated such as diamond [10], circular, elliptical and drop [11], helical and continuous [12], angled [13], twisted [14], delta [15], trapezoidal winglet [16], arc [17], 'L' [18], U' [19], 'V' [20], multiple 'V' [21], perforated ' $\mathrm{V}$ ' [22], double 'V' [23], discrete ' $\mathrm{W}$ ' [24], and 'Z'-shaped [25].

The main objective of the present computational fluid dynamic study is to examine the turbulent flow of air inside an isothermal wall channel of rectangular section, containing four flat rectangular baffle plates, as presented in more detail in Demartini et al. [3]. The geometry of the problem is a simplification of the geometry of baffle plates found in shell-and-tube heat exchangers [3]. This is an important problem in the scope of shelland-tube heat exchangers where the characterization of the fluid flow, temperature distribution as well as the existence and the extension of possible vortices need to be identified [3]. The impact of the flow rate is studied by varying Re from 12,000 to 21,000 with an increment of 3,000 .

\section{PHYSICAL MODEL AND TURBULENCE MODELLING Problem Definition}

The system of interest is a two-dimensional channel with four staggered, transverse baffle plates and a constant temperature along both walls as shown in Figure 1. The computational domain is a $0.858 \mathrm{~m}$ long rectangular duct $(\mathrm{L}), 0.146 \mathrm{~m}$ height $(\mathrm{H})$, with $0.193 \mathrm{~m}$ width $(\mathrm{W})$ and an aeraulic diameter $\left(\mathrm{D}_{\mathrm{h}}\right)$ of $0.167 \mathrm{~m}$. This channel is divided into three sections: inlet $\left(\mathrm{L}_{\text {in }}=0.218 \mathrm{~m}\right)$, between baffle plates $(\mathrm{Pi}=0.142 \mathrm{~m})$ and exit $\left(\mathrm{L}_{\text {out }}=\right.$ $0.174 \mathrm{~m}$ ). The distance between the top edge of the baffle plate and the wall (h) was kept constant at $0.08 \mathrm{~m}$. This corresponds to the area reduction of $54.794 \%$ at the baffle edge. The thickness of baffle plates (w) is set to 0.01 
m. The aspect ratio of channel width-to-height, channel length-to-hydraulic diameter, baffle spacing-to-channel height ratio, and blockage ratio of baffle height-to-channel height are fixed at $\mathrm{W} / \mathrm{H}=1.321, \mathrm{~L} / \mathrm{D}_{\mathrm{h}}=5.137, \mathrm{Pi} / \mathrm{H}=$ 0.972 , and $\mathrm{h} / \mathrm{H}=0.547$, respectively. Air is the working fluid with the flow rate in terms of Reynolds number ranging from 12,000 to 21,000 .

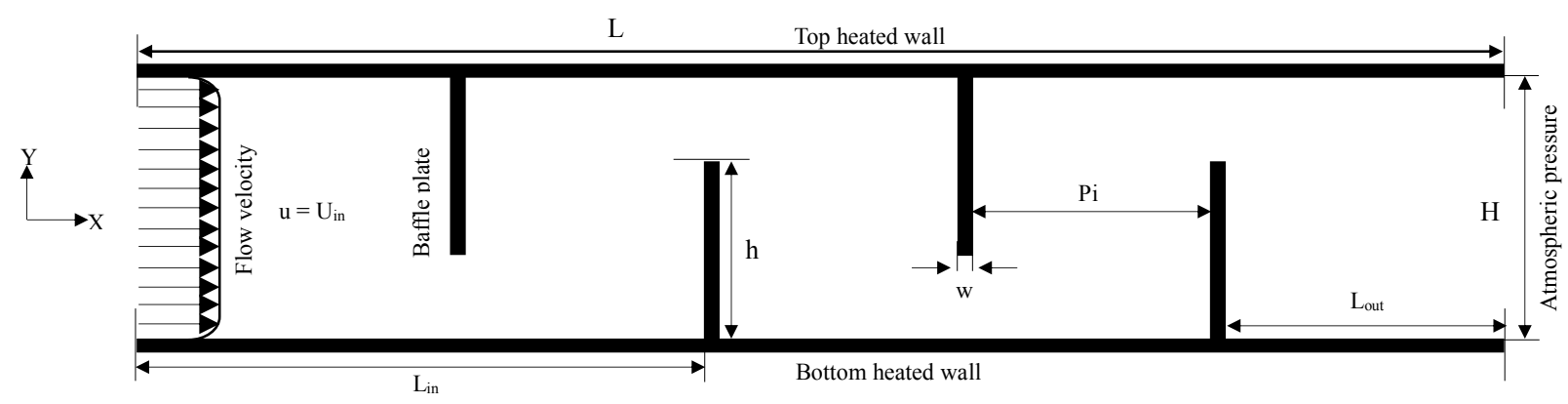

Figure 1. Computational domain with boundary conditions

The assumptions of the whole domain investigated for this work are as follows:

- Steady two-dimensional fluid flow and heat transfer

- The flow is turbulent and incompressible

- Constant fluid properties

- $\quad$ The top and bottom wall temperature is fixed

- Body forces and viscous dissipation are ignored

- Negligible radiation heat transfer

- $\quad$ An eddy viscosity model was used to account for the effect of turbulence phenomena

\section{Governing Equations}

Based on the above assumptions, the duct flow model is governed by the Reynolds averaged NavierStokes (RANS) equations with the k- $\varepsilon$ turbulence model [26] and the energy equation. Then, the governing equations can be written as

Continuity equation:

$$
\frac{\partial}{\partial \mathrm{x}_{\mathrm{i}}}=\left(\rho \mathrm{u}_{\mathrm{i}}\right)=0
$$

Momentum equation:

$$
\frac{\partial}{\partial \mathrm{x}_{\mathrm{i}}}\left(\rho \mathrm{u}_{\mathrm{i}} \mathrm{u}_{\mathrm{j}}\right)=\frac{\partial}{\rho \mathrm{x}_{\mathrm{i}}}\left[\mu\left(\frac{\partial \mathrm{u}_{\mathrm{i}}}{\partial \mathrm{x}_{\mathrm{j}}}-\rho \overline{\mathrm{u}_{\mathrm{l}}^{\prime} \mathrm{u}_{\mathrm{j}}^{\prime}}\right)\right]-\frac{\partial \mathrm{P}}{\partial \mathrm{x}_{\mathrm{i}}}
$$

where $\rho$ is the fluid density, $P$ the pressure, $\mu$ dynamic viscosity, $u_{i}$ and $u_{j}$ are mean velocity components in $x_{i}$ and $\mathrm{x}_{\mathrm{j}}$ directions.

Energy equation:

$$
\frac{\partial}{\partial \mathrm{x}_{\mathrm{i}}}\left(\rho \mathrm{u}_{\mathrm{i}} \mathrm{T}\right)=\frac{\partial}{\rho \mathrm{x}_{\mathrm{i}}}\left(\left(\Gamma+\Gamma_{\mathrm{t}}\right) \frac{\partial \mathrm{T}}{\partial \mathrm{x}_{\mathrm{j}}}\right)
$$

where $\Gamma$ and $\Gamma_{\mathrm{t}}$ are molecular thermal diffusivity and turbulent tharmal diffusivity, repectively and are given by

$$
\Gamma=\mu / \operatorname{Pr} \text { and } \Gamma_{\mathrm{t}}=\mu_{\mathrm{t}} / \operatorname{Pr}_{\mathrm{t}}
$$


In equation 2, $\rho \overline{\mathrm{u}_{\mathrm{l}}^{\prime} \mathrm{u}_{\mathrm{j}}^{\prime}}$ is the Reynolds stresses defined by the Boussinesq hypothesis as

$$
-\rho \overline{u_{1}^{\prime} u_{j}^{\prime}}=\mu_{t}\left(\frac{\partial u_{i}}{\partial x_{j}}+\frac{\partial u_{j}}{\partial x_{i}}\right)-\frac{2}{3}\left(\rho \mathrm{k}+\mu_{t} \frac{\partial u_{i}}{\partial x_{j}}\right) \delta_{i j}
$$

where $\delta_{\mathrm{ij}}$ is the Kroenecker delta and $\mu_{\mathrm{t}}$ the eddy viscosity defined as

$$
\mu_{\mathrm{t}}=\rho \mathrm{C}_{\mu} \frac{\mathrm{k}^{2}}{\varepsilon}
$$

The standard k- $\varepsilon$ model, based on Launder and Spalding [26], is defined by two transport equations, one for the turbulent kinetic energy, $\mathrm{k}$ and the other for the specific dissipation rate $\varepsilon$, as given below

$$
\begin{gathered}
\frac{\partial}{\partial \mathrm{x}_{\mathrm{j}}}\left(\rho \mathrm{ku}_{\mathrm{j}}\right)=\frac{\partial}{\partial \mathrm{x}_{\mathrm{j}}}\left[\left(\mu+\frac{\mu_{\mathrm{t}}}{\sigma_{\mathrm{k}}}\right) \frac{\partial \mathrm{k}}{\partial \mathrm{x}_{\mathrm{j}}}\right]+\mathrm{G}_{\mathrm{k}}+\rho \varepsilon \\
\frac{\partial}{\partial \mathrm{x}_{\mathrm{j}}}\left(\rho \varepsilon \mathrm{u}_{\mathrm{j}}\right)=\frac{\partial}{\partial \mathrm{x}_{\mathrm{j}}}\left[\left(\mu+\frac{\mu_{\mathrm{t}}}{\sigma_{\varepsilon}}\right) \frac{\partial \varepsilon}{\partial \mathrm{x}_{\mathrm{j}}}\right]+\mathrm{C}_{1 \varepsilon} \frac{\varepsilon}{\mathrm{k}}-\mathrm{C}_{2 \varepsilon} \rho \frac{\varepsilon^{2}}{\mathrm{k}}
\end{gathered}
$$

In equations 7 and $8, \mathrm{G}_{\mathrm{k}}$ represents the production rate of the kinetic energy due to the energy transfer from the mean flow to turbulence.

The empirical constants for the standard k- $\varepsilon$ model are assigned the following values [26]:

$$
\mathrm{C}_{\mu}=0.99, \mathrm{C}_{1 \varepsilon}=1.44, \mathrm{C}_{2 \varepsilon}=1.92, \sigma_{\mathrm{k}}=1.0, \sigma_{\varepsilon}=1.3
$$

\section{Governing Parameters}

The flow Reynolds number ( $\mathrm{Re}$ ) based on channel aeraulic diameter

$$
\mathrm{D}_{\mathrm{h}}=2 \mathrm{HW} /(\mathrm{H}+\mathrm{W})
$$

is given by

$$
\operatorname{Re}=\rho \bar{U} D_{\mathrm{h}} / \mu
$$

The skin friction coefficient $\left(\mathrm{C}_{\mathrm{f}}\right)$ is given by

$$
\mathrm{C}_{\mathrm{f}}=2 \tau_{\mathrm{w}} / \rho \overline{\mathrm{U}}^{2}
$$

The friction factor $(\mathrm{f})$ is evaluated from the pressure drop $(\Delta \mathrm{P})$ as

$$
\mathrm{f}=2(\Delta \mathrm{P} / \mathrm{L}) \mathrm{D}_{\mathrm{h}} / \rho \overline{\mathrm{U}}^{2}
$$

where $\bar{U}$ presents the average axial velocity of the section, and $\tau_{\mathrm{w}}$ is the shear stress to the wall.

For determining the heat transfer rate inside the channel, the heat transfer is measured by the local Nusselt number $\left(\mathrm{Nu}_{\mathrm{x}}\right)$ which can be written as

$$
\mathrm{Nu}_{\mathrm{x}}=\mathrm{h}_{\mathrm{x}} \mathrm{D}_{\mathrm{h}} / \mathrm{k}_{\mathrm{f}}
$$

and the average Nusselt number $(\mathrm{Nu})$ can be obtained by

$$
\mathrm{Nu}=\frac{1}{\mathrm{~L}} \int \mathrm{Nu}_{\mathrm{x}} \partial \mathrm{x}
$$


The following expression represents the thermal enhancement factor (TEF):

$$
T E F=\left(N u / N u_{0}\right) /\left(f / f_{0}\right)^{1 / 3}
$$
respectively.

The quantities $\mathrm{Nu}_{0}$ and $\mathrm{f}_{0}$ are the average Nusselt number and the friction factor of the smooth channel,

The Dittus and Boelter correlation has the form [27]:

$$
N u_{0}=0.023 \operatorname{Re}^{0.8} \operatorname{Pr}^{0.4} \text { for } \operatorname{Re} \geq 10^{4}
$$

The Petukhov correlation has the form [27]:

$$
f_{0}=(0.79 \ln \operatorname{Re}-1.64)^{-2} \text { for } 3 \times 10^{3} \leq \operatorname{Re} \leq 5 \times 10^{6}
$$

\section{Boundary Conditions}

The aerodynamic boundary conditions are set according to the numerical and experimental data of Demartini et al. [3] while the thermal boundary conditions are chosen according to the numerical study of Nasiruddin and Kamran Siddiqui [5]. For full height baffled channel, a uniform one-dimensional velocity profile is introduced as the aeraulic boundary condition [3] at the inlet of the computational domain while a pressure outlet condition is applied at the exit, as shown in Figure 1. The temperatures of the entire wall of the test section $\left(\mathrm{T}_{\mathrm{w}}\right)$ and inlet fluid $\left(\mathrm{T}_{\text {in }}\right)$ are set as constant, which are respectively $375 \mathrm{~K}$ and $300 \mathrm{~K}$, as the thermal boundary condition [5]. In the k- $\varepsilon$ model, the near-wall region was simulated by a two zone model, i.e. viscous sub layer and fully turbulent zone, and the wall function suggested by Laundry and Spalding [26] was used.

\section{COMPUTATIONAL FLUID DYNAMIC SIMULATION Numerical Schemes}

A non-uniform grid system with a large concentration of nodes in regions of steep gradients, such as those close to the walls and baffle plates was employed, as shown in Figure 2. The wall functions given by Laundry and Spalding [26] are employed to prescribe the boundary conditions along the faces of the baffle plates and the channel walls in the computational domain. In this study, CFD modelling is performed using the commercial CFD software FLUENT 6.3. The finite volume method, developed by Patankar [28], is adopted for the discretization, and all the variables are treated with the Quick scheme in the form given by Leonard and Mokhtari [29], except the pressure term with Second order upwind scheme [28]. The SIMPLEC discretization algorithm was used to solve the pressure-velocity coupling in order to avoid the appearance of irrational pressure field and velocity field, details of which can be found in Van Doormaal and Raithby [30].

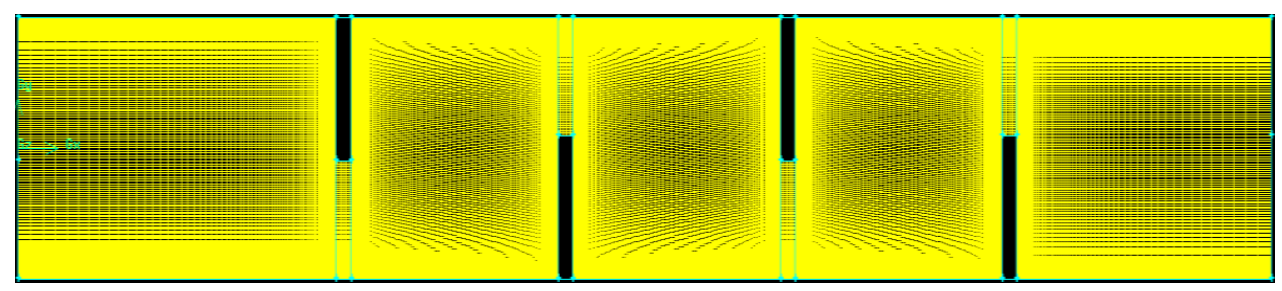

Figure 2. Mesh system with refinements near the all solid boundaries

The numerical solution is assumed to converge when the relative error on $\phi$ is smaller than $\delta$ as

$$
\max \left(\frac{\left|\phi-\phi^{*}\right|}{\left|\phi^{*}\right|}\right)<\delta
$$

where ${ }^{*}$ denote values of the previews iterations, $\phi$ represents the dependent variables of $\mathrm{u}, \mathrm{v}, \mathrm{k}, \varepsilon$ and $\mathrm{T}$, and $\delta$ is a prescribed error. Here, we select $\delta=10^{-9}$ for $\phi \equiv(\mathrm{u}, \mathrm{v}, \mathrm{k}$, and $\varepsilon)$, and $\delta=10^{-12}$ for $\phi \equiv(\mathrm{T})$. 


\section{Grid Independence}

The effect of the grid size on the mean axial velocity and average Nusselt number was studied by employing different mesh sizes for the conditions of $\mathrm{W} / \mathrm{H}=1.321, \mathrm{~L} / \mathrm{D}_{\mathrm{h}}=5.137, \mathrm{Pi} / \mathrm{H}=0.972, \mathrm{~h} / \mathrm{H}=0.547$ at $\mathrm{Re}=8.73 \times$ $10^{4}$ as shown in Table 1.

Table 1. Effect of grid size on CFD solution for $\mathrm{W} / \mathrm{H}=1.321, \mathrm{~L} / \mathrm{D}_{\mathrm{h}}=5.137, \mathrm{Pi} / \mathrm{H}=0.972, \mathrm{~h} / \mathrm{H}=0.547$ at $\operatorname{Re}=8.73 \times 10^{4}$

\begin{tabular}{|c|c|c|}
\hline Grid & $\mathbf{U}_{\max }(\mathbf{m} / \mathbf{s}) \mathbf{a t} \mathbf{x}=\mathbf{0 . 8 2 9} \mathbf{~ m}$ & $\mathbf{N u}$ at $\mathbf{y}=\mathbf{H} / \mathbf{2}$ \\
\hline $220 \times 45$ & 41.370472 & 4049.527958900 \\
\hline $270 \times 65$ & 41.622475 & 4082.754851400 \\
\hline $302 \times 85$ & 41.748477 & 4111.828387333 \\
\hline $370 \times 105$ & 41.794677 & 4153.241552455 \\
\hline $420 \times 135$ & 42.000780 & 4153.362474412 \\
\hline
\end{tabular}

It is found that the variation in $U_{\max }$ and $\mathrm{Nu}$ is marginal when increasing the number of nodes from 370 $\times 105$ to $420 \times 135$ (in $X$ and $Y$ directions respectively). The minimum relative error is about $0.5 \%$ on $U_{\max }$ value and less than $0.0029 \%$ on Nu value. Hence, the grid size of $370 \times 105$ was adopted for the current simulation.

\section{Model Validation}

In order to verify the accuracy of the proposed CFD model used in this study, the obtained results were compared in terms of pressure and velocity profiles with the numerical and experimental data obtained from Demartini et al. [3] for the same configuration, system properties and operating conditions, for $\mathrm{W} / \mathrm{H}=1.321, \mathrm{~L} / \mathrm{D}_{\mathrm{h}}$ $=3.317, \mathrm{Pi} / \mathrm{H}=0.972, \mathrm{~h} / \mathrm{H}=0.547$ at $\mathrm{Re}=8.73 \times 10^{4}$. Both the numerical and experimental profiles of dynamic pressure and axial velocity are presented and computed along the height of the channel at transverse axial stations $\mathrm{x}=0.223 \mathrm{~m}$ and $\mathrm{x}=0.525 \mathrm{~m}$, as shown in Figure $3(\mathrm{a}$ and $\mathrm{b}$ ), respectively.

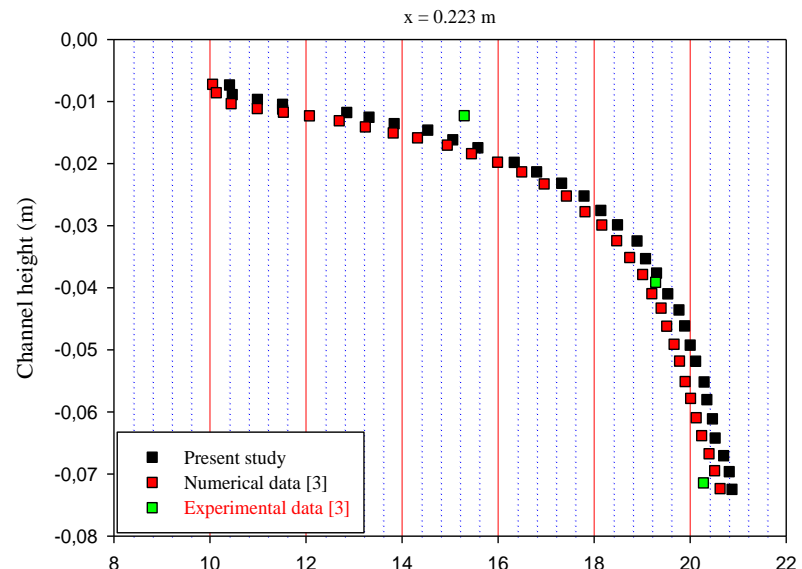

a)

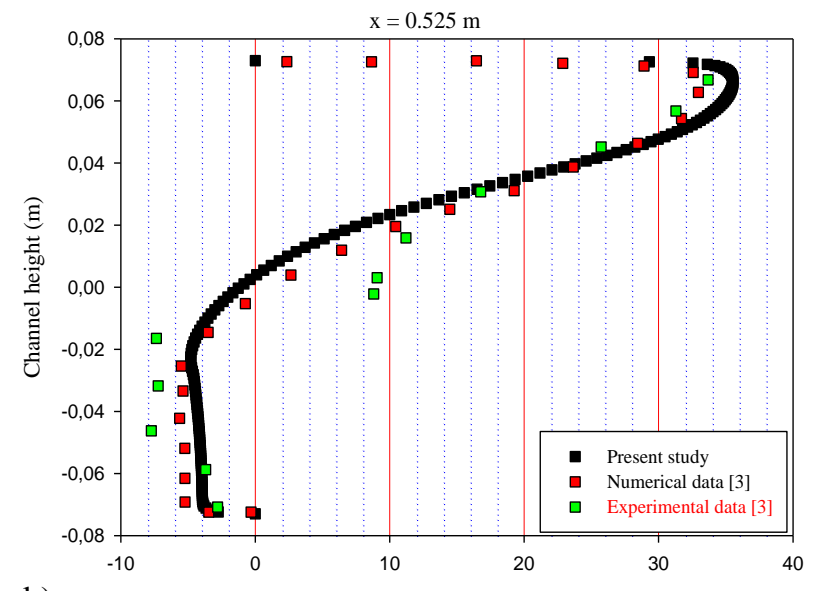

b)

Axial velocity $(\mathrm{m} / \mathrm{s})$

Figure 3. Comparison of numerical and experimental profiles in terms of a) dynamic pressure and b) axial velocity for $\mathrm{Re}=8.73 \times 10^{4}$

As can be seen from the figure, there is a quite acceptable agreement between the numerical and experimental data. Thus, it was concluded that the present CFD simulation, accurately predicts the air flow comportment within the given channel.

The variation of Nusselt number with Re value is also presented for a smooth rectangular air channel and compared with empirical correlation of Dittus-Boelter [27]. As seen in Figure 4, there is a good agreement between the present numerical smooth channel results and the correlation solutions in the open literature [27]. These results give confidence that the computational approach used was accurate. 


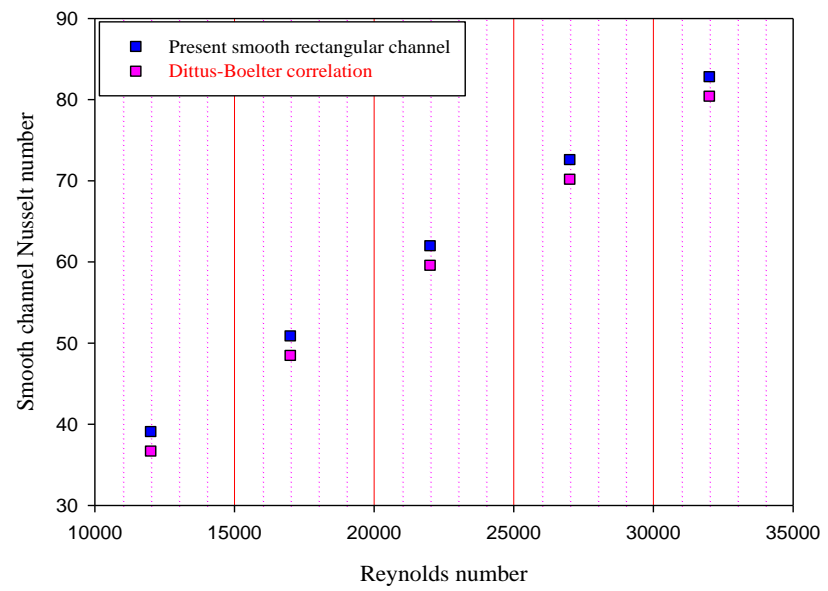

Figure 4. Verification of Nusselt number for smooth rectangular channel

\section{RESULTS AND DISCUSSION}

\section{Flow Topology and Temperature Field}

The impact of the flat rectangular baffle plates on the structure of the near-wall flow could be easily discerned by considering the streamlines, axial velocity, dynamic pressure, turbulent kinetic energy, turbulent viscosity, and temperature fields, as shown respectively in Figures 5-9 for the cases of $\mathrm{W} / \mathrm{H}=1.321, \mathrm{~L} / \mathrm{D}_{\mathrm{h}}=5.137$, $\mathrm{Pi} / \mathrm{H}=0.972$, and $\mathrm{h} / \mathrm{H}=0.547$. Here the flow field contours around the baffle plates are presented for Reynolds numbers of $12,000,15,000,18,000$, and 21,000 .

Figure 5, corresponding to the case of streamlines, shows that the airflow is disturbed by the presence of these deflectors that are staggering arranged on both the heated top and bottom walls of the channel.

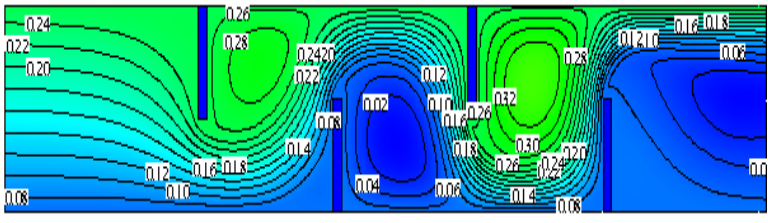

a)

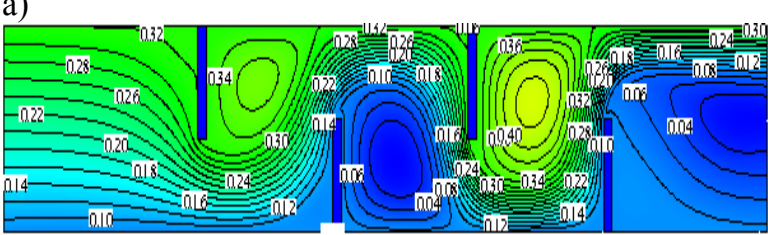

b)

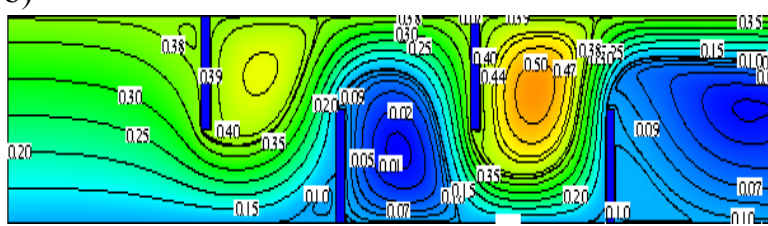

c)

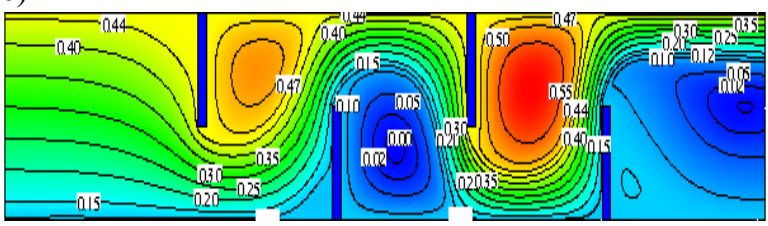

d)

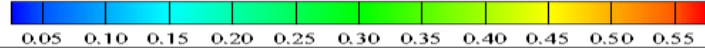

Figure 5. Distributions of streamlines

in

the whole domain examined for a) $\operatorname{Re}=12,000$, b) $\operatorname{Re}=15,000$, c) $\operatorname{Re}=18,000$, and d) $\operatorname{Re}=21,000$

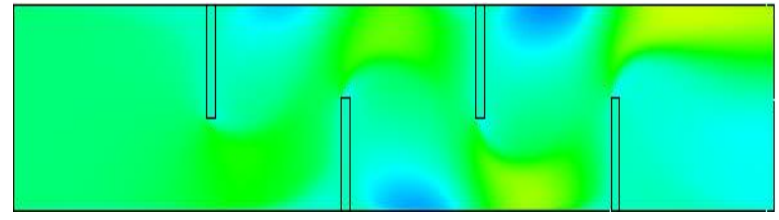

a)

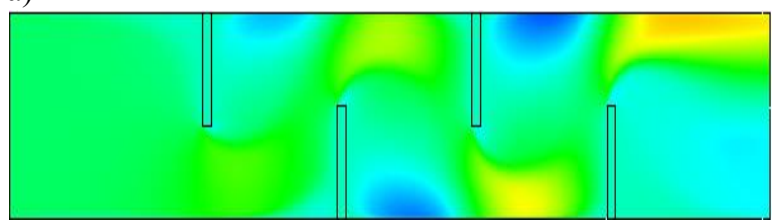

b)

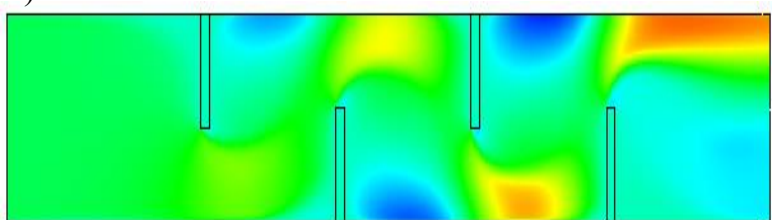

c)

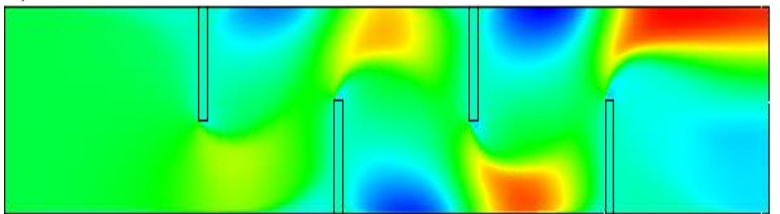

d)

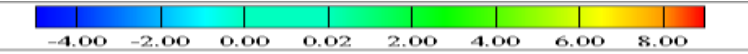

Figure 6. Distributions of axial velocity fields in the whole domain investigated for a) $R e=12,000$, b) $\operatorname{Re}=15,000, \mathrm{c}) \mathrm{Re}=18,000$, and d) $\mathrm{Re}=21,000$ 
In all cases, a very small vortex is observed in the vicinity of the upper left corner of the first baffle plate, which was induced due to the flow separation. Downstream of the considered baffle plate, as a result of sudden expansion in the cross, the air flow separates, a strong flow recirculation zone is observed on the back side of the first upper wall-mounted baffle plate and air flow reattachment is the established. This phenomenon is cause by the insertion of the transverse plane baffles into the channel, leading to a backward-facing step flow field. The size of this vortex is found to be larger than the one at the left corner and its height was approximately equal to extent of the flow blockage $(\mathrm{h} / \mathrm{H})$ by the baffle plate, which is equal to 0.547 for the investigated baffle case. This corresponds to the area reduction of $54.794 \%$ at the baffle plate edge. As seen from this figure, the backwardfacing step flow field causes the fluid to strongly rotating motion, which enhances mixing by brings the parcels of air from the near-wall area to the bulk and vice versa. Similar behaviors are observed in the regions near to the other lower and upper wall-mounted deflectors with vortices at the front of the baffle plates and back areas. These observations are confirmed by Demartini et al. [3], Nasiruddin and Kamran Siddiqui [5], and Mohammadi Pirouz et al. [32]. The structure of fluid flow using various values of Reynolds number looks similar except for flow regions around the deflector corners.

Figure 6 shows the axial velocity field distribution on the longitudinal section. At Reynolds numbers varied from 12,000 to 21,000, the air flows under, above and between the baffle plates by taking their exact shape with the presence of recirculation zones upstream and downstream from each deflector. Due to the changes in the air flow direction produced by these baffle plates, the velocity near the deflector walls is very low, and the velocity near the channel walls is very high with an acceleration process that starts just before the plates. The velocity of air flow in the recirculation regions at shear layer side is slower, or even presents a negative velocity value, while that at the free stream side is a positive velocity, showing a significant difference of velocity at both sides, similar to the experimental data reported by Lin [33] for the case of a two-dimensional incompressible flow of a Newtonian fluid inside a rectangular-channel with various types of transverse baffles. The fluid velocity increases with the rise of Reynolds number $(\mathrm{Re})$ and thus, the $\mathrm{Re}=21,000$ provides maximum air velocity.

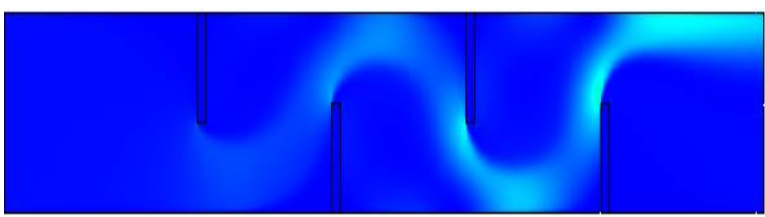

a)

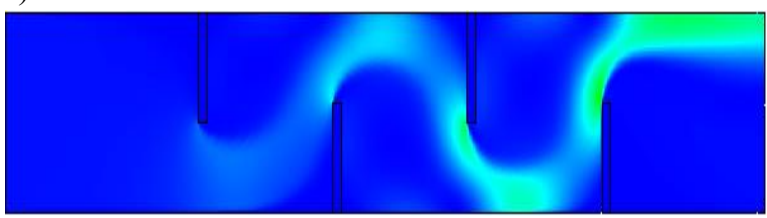

b)

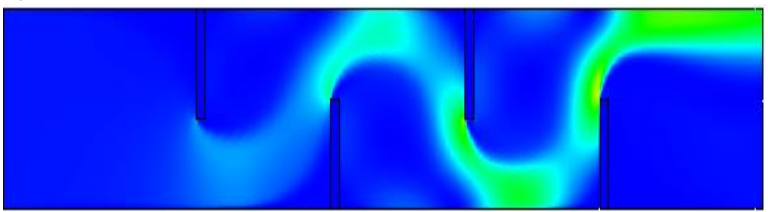

c)

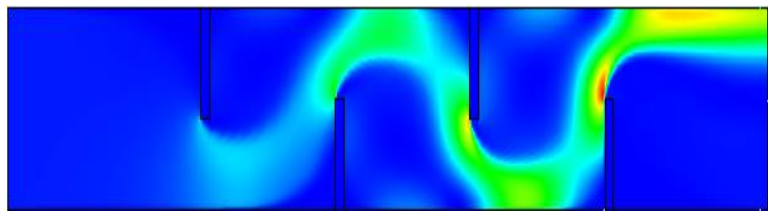

d)

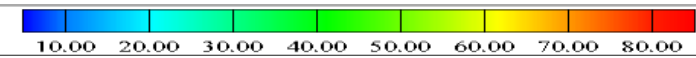

Figure 7. Distributions of dynamic pressure fields in the whole domain treated for a) $\mathrm{Re}=12,000$,

b) $\operatorname{Re}=15,000, \mathrm{c}) \mathrm{Re}=18,000$, and d) $\mathrm{Re}=21,000$

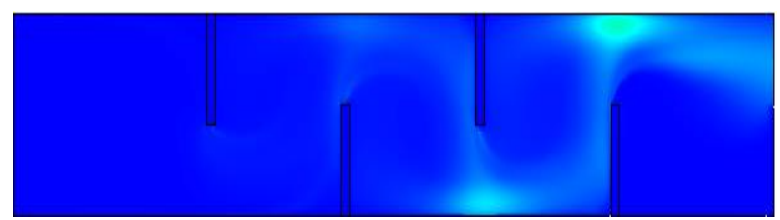

a)

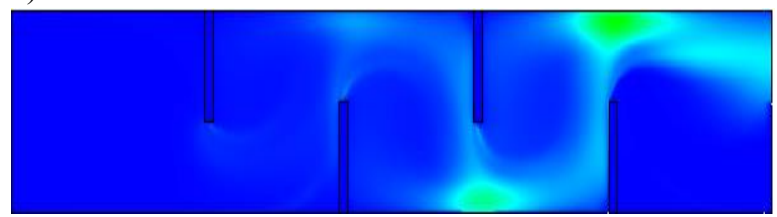

b)

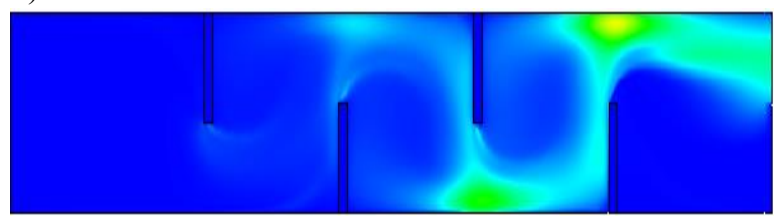

c)

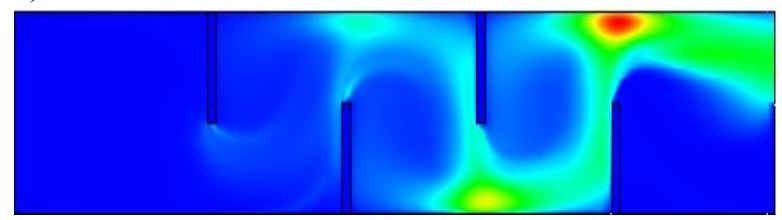

d)

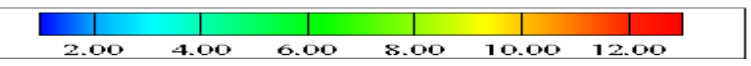

Figure 8. Distributions of turbulent kinetic energy fields in the whole domain analyzed for a) $\mathrm{Re}=12,000$, b) $\operatorname{Re}=15,000, \mathrm{c}) \mathrm{Re}=18,000$, and d) $\mathrm{Re}=21,000$ 
The contour plots of dynamic pressure fields are displayed in Figure 7 for different Reynolds number values, $\operatorname{Re}=12,000-21,000$. The largest variations in the dynamic pressure occur in the regions near to the upstream and top faces of the baffle plates, as expected. In the figure, it is visible that the fluid dynamic pressure in the locations corresponding to the zones of recirculation is significantly low as compared to those in the same region of no baffle plate case. However, the dynamic pressure values are increased again at the area of high velocity especially in the regions opposite the baffle plate tips. Due to the deviations in the flow direction produced by these deflectors, the highest dynamic pressure values appear near the top heated channel wall with an increase in the flow momentum that starts just before the baffle plates. The figure also shows that the dynamic pressure coefficient increases with an increase in the Reynolds number.

The variation of turbulent kinetic energy (TKE) is presented in Figure 8 which shows that the TKE increases with the increase of Reynolds number. The peak TKE values are shown in the region opposite the baffle tip near the channel walls, while the TKE is observed to be very low at the areas corresponding to the zones of vortex as seen in the figure. A large TKE zone is found in the last baffle plate regime close to the top channel wall which yields the strong influence of turbulence intensity on heat transfer enhancement.

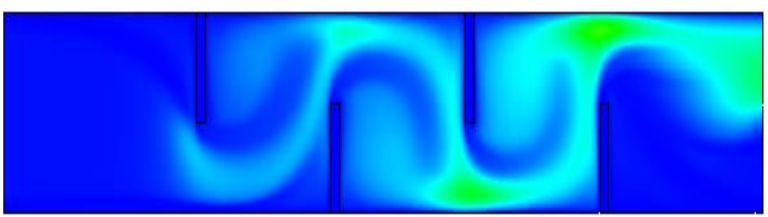

a)

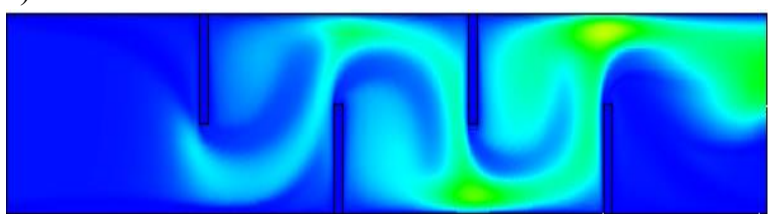

b)

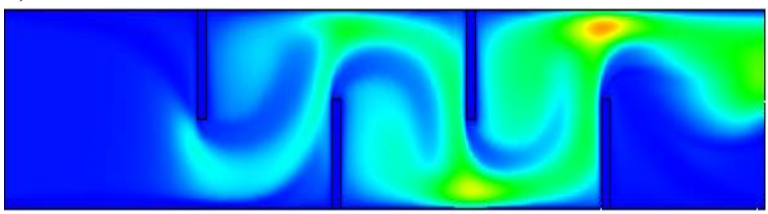

c)

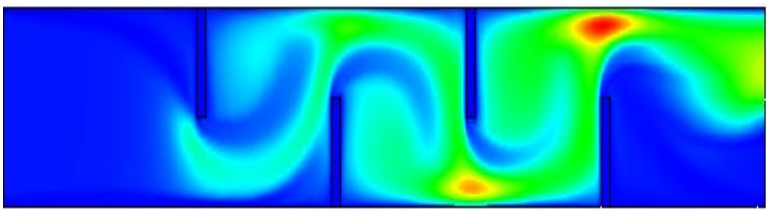

d)

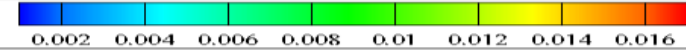

Figure 9. Distributions of turbulent viscosity fields in the baffled channel for a) $\mathrm{Re}=12,000$, $\operatorname{Re}=15,000, c) \operatorname{Re}=18,000$, and d) $\operatorname{Re}=21,000$

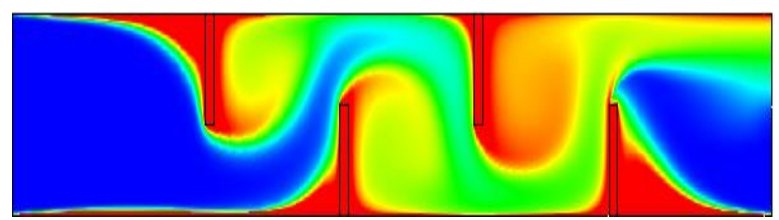

a)

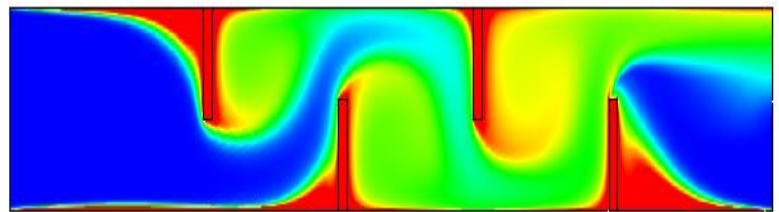

b)

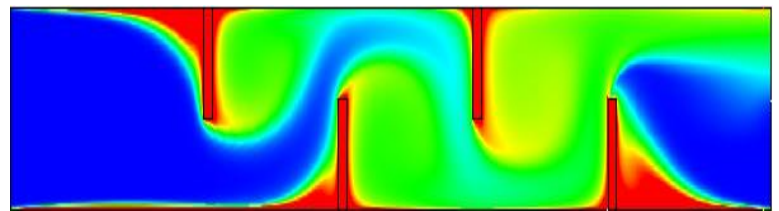

c)

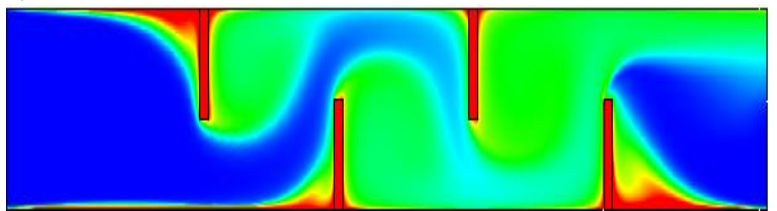

d)

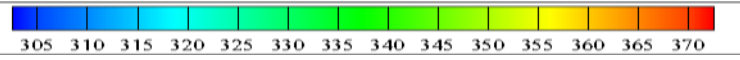

Figure 10. Distributions of temperature fields in the baffled channel for a) $\operatorname{Re}=12,000, b) \operatorname{Re}=15,000$,

c) $\operatorname{Re}=18,000$, and d) $\operatorname{Re}=21,000$

Figure 9, presents the turbulent viscosity field contour plots in the rectangular-channel for using the baffle plates with various Reynolds number values. It is seen in this figure that the baffle plates can induce longitudinal recirculation flows in the lower and upper parts along the channel, leading to higher turbulence viscosity. In addition, the acceleration and expansion of the air are clear when it flows cross baffle plates, and the flow direction varies, which generates multidirectional jet and results in the turbulent viscosity enhancement. This is because that the airflow area decreases $54.794 \%$ and the velocity increase substantially. Similarly to the results in Figure 7, it is apparent that the high viscosity values are seen in large areas over the channel walls, especially in the regions between the tip of the baffle plates and the channel walls. However, the air flow pattern has no significant effect on the left face area of the left top wall-mounted deflector and on the right face area of the right bottom wallattached turbulator, which is due to deviation in flow direction with large velocity in those regions. These same 
contour plots also indicate that the turbulent viscosity values increase considerably as the number of Reynolds increases.

The contour plots of temperature fields along the constant temperature-surfaced rectangular channel are shown in Figure 10. As seen from the figure, the higher temperature gradient can be observed where the air flow impinges the channel wall while the lower one is found at the baffle plate corner area where the corner recirculation zone occurs, especially area behind the deflectors. This comportment shows the same behavior as results of Nasiruddin and Kamran Siddiqui [5], Sripattanapipat and Promvonge [10], and Sriromreun et al. [25]. These results do confirm the fact that the heat exchange between the fluid and the hot wall, for our study, is more important when low Reynolds numbers are used. Moreover, based on the analysis of the numerical results of the axial velocity fields (see Figure 6), as well as that of the fluid temperature fields (see Figure 10), for different regions of the channel, a relation exists between the fluid temperature and the flow velocity. There is an inverse proportionality between increasing Reynolds number and the fluid temperature in each channel station.

\section{Axial Velocity Profiles in Specific Sections of the Channel}

The axial velocity profiles are also affected by the Reynolds number as it appears in Figure 11. Increasing Re from 12,000 to 21,000, when $\mathrm{W} / \mathrm{H}, \mathrm{L} / \mathrm{D}_{\mathrm{h}}, \mathrm{Pi} / \mathrm{H}$, and $\mathrm{h} / \mathrm{H}$ are kept at constant values, leads to a disruption of the flow and the formation of a recirculation zone behind each deflector. The negative velocities indicate the presence of recirculation cells behind the baffles. These recirculation zones increase in size and occupy more and more the area between the deflectors while going towards the high values of Re due to the increase in the inlet velocity.
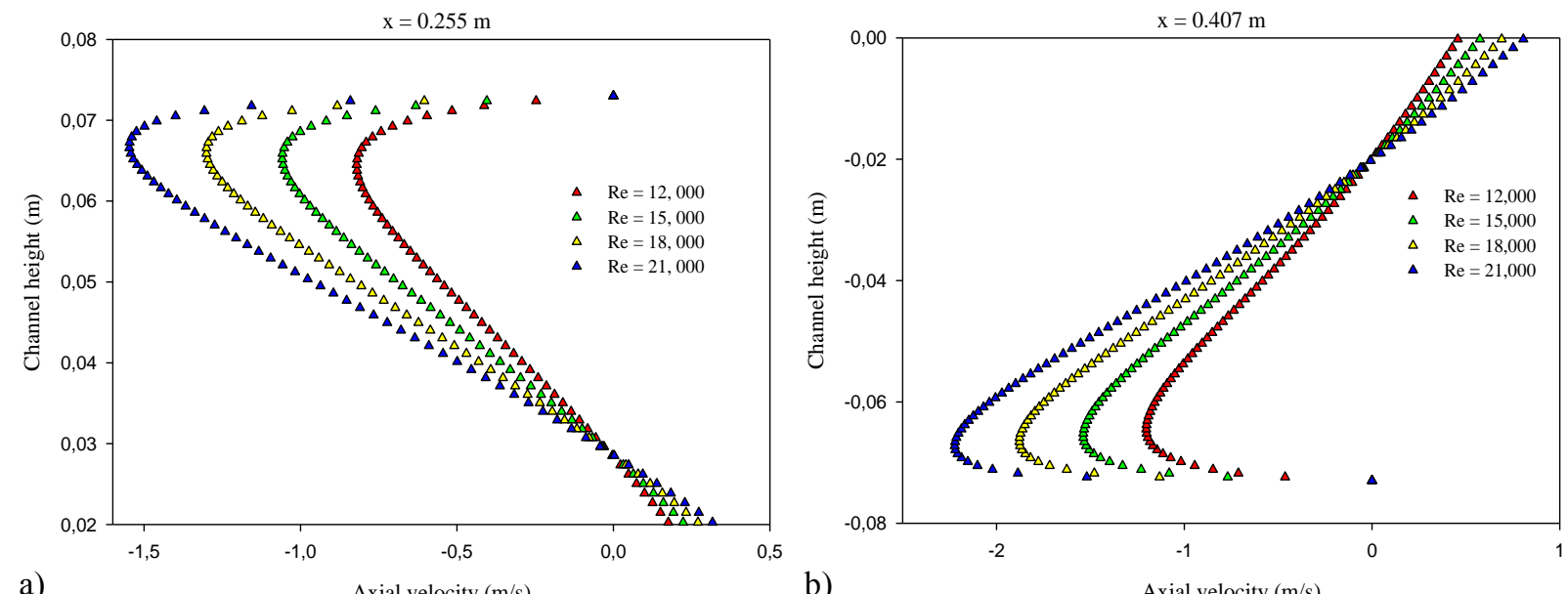

a)

b)

Axial velocity $(\mathrm{m} / \mathrm{s})$

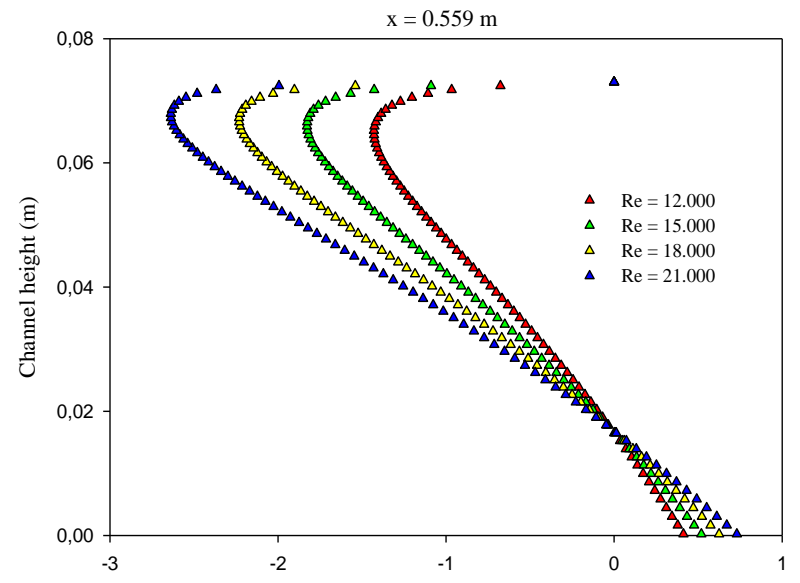

c)

Axial velocity (m/s)

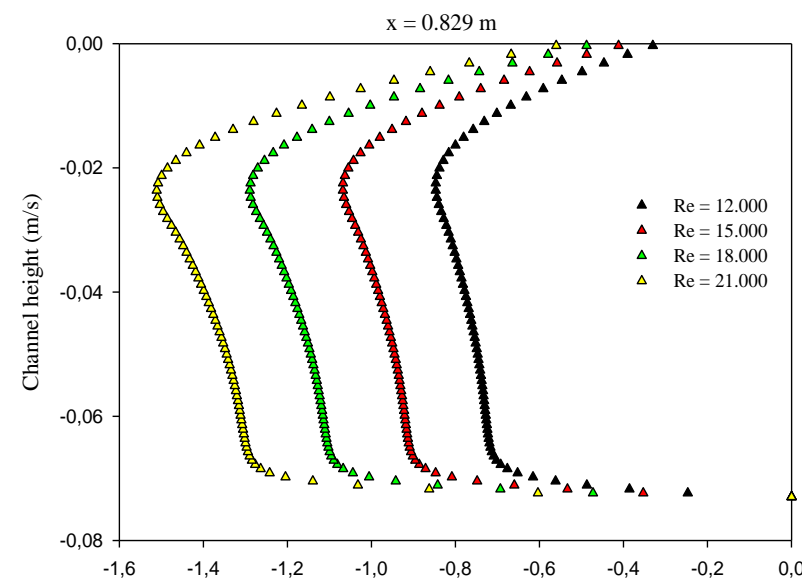

d)

Axial velocity $(\mathrm{m} / \mathrm{s})$

Figure 11. Comparison of the vortex length for various Re values just downstream the (a) left upper wallmounted turbulator at $\mathrm{x}=0.255 \mathrm{~m}$, (b) left lower wall-attached deflector at $\mathrm{x}=0.407 \mathrm{~m}$, (c) right top wall-fixed disturbance promoter at $\mathrm{x}=0.559 \mathrm{~m}$, and (d) right bottom-placed baffle at $\mathrm{x}=0.829 \mathrm{~m}$ 
In addition, the variation in the flow rates in terms of Re number have no effect of the flow pattern, but results in the level of the recirculation length. It is noted that both the velocity and length of these vortices tend to increase with the rise of Re number values. Behind the baffles, at positions given by $\mathrm{x}=0.255,0.407,0.559$ and $0.829 \mathrm{~m}, 0.027 \mathrm{~m}$ after each deflectors, the value of the axial velocity at the recirculation locations reaches respectively $-2.2,-2.2,-2.6$ and $-1.5 \mathrm{~m} / \mathrm{s} ; 0.835,0.835,0.706$, and 1.225 times lower than the entrance velocity, which is $1.8375 \mathrm{~m} / \mathrm{s}$ at high Reynolds number value, $\mathrm{Re}=21,000$, as shown in the figure.

The effect of the flow Reynolds number variation on the distribution of axial velocity profiles through the channel in the positions starting from the tip of the baffles to the opposite walls at stations given by $x=0.223 \mathrm{~m}$, $0.375 \mathrm{~m}$, and $0.527 \mathrm{~m}$ from the entrance, for the cases of the investigated configuration, is displayed in figure 10 (a to c), respectively, and four values of $\operatorname{Re}$ are considered: $\operatorname{Re}=12,000 ; 15,000 ; 18,000$, and $\operatorname{Re}=21,000$. As expected, obviously it can be observed that values of axial velocity become higher with increasing values in flow Reynolds number for all stations proposed.

\section{Skin Friction Loss}

Figure 12 (a) shows the variations in the normalized skin friction coefficient $\left(\mathrm{Cf} / \mathrm{f}_{0}\right)$ using the standard kepsilon turbulence model in the range of Reynolds number investigated, $R e=12,000-21,000$, for fixed values of other parameters. $\mathrm{f}_{0}$ is the friction factor for the smooth air channel at the same Reynolds number, and is given by equation (18). The trends of skin friction are similar for all Reynolds number values. The friction coefficients show the highest value in the zones opposite the lower wall-mounted deflectors and the lowest value in the regions around the upper wall-attached deflectors for all Re values. The influence of the Reynolds number on the profiles of the local coefficient of normalized skin friction along the top channel wall is also shown in this same figure. Changes for Reynolds numbers ranging from 12,000 to 21,000, we noted that the increase in the Reynolds number changes the coefficient of skin friction significantly. These results are obtained because the increase in the Reynolds number increases the flow rate of introducing large areas of recycling, which leads to an acceleration of the air flow and also the coefficient of friction and consequently pressure drop. It addition, these observations are confirmed by further evolution of the coefficient of mean friction, as shown in Figure 12 (b).
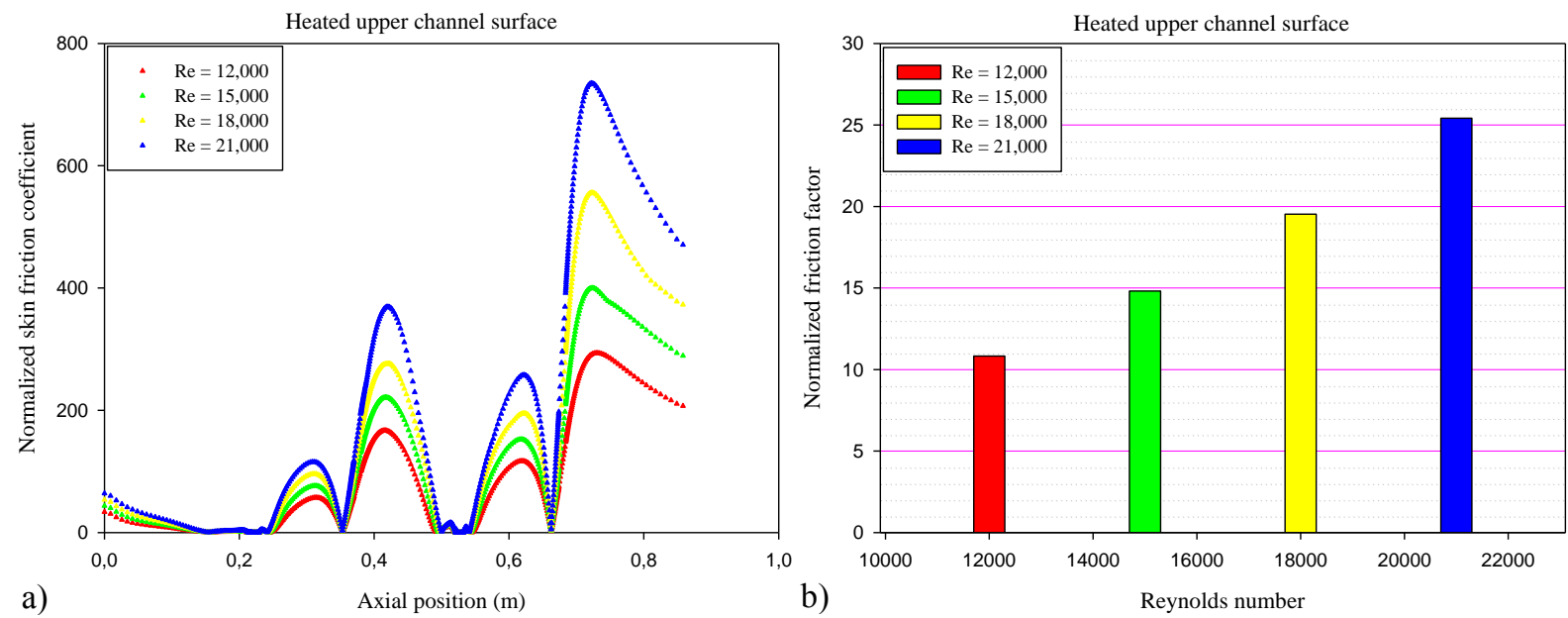

Figure 12. Variations of normalized a) local and b) average friction coefficients with Reynolds numbers

Figure 12 (b) illustrates the variation of the normalized average skin friction coefficient $\left(\mathrm{f} / \mathrm{f}_{0}\right)$ calculating along the top channel wall, corresponding to transverse position $\mathrm{y}=\mathrm{H} / 2 \mathrm{~m}$, and for a Reynolds number range from 12,000 to 21,000 . It is clear that there is direct proportionality between the increase in the coefficient of mean friction and the elevation of the Reynolds number. The maximum friction factor is obtained for the highest Reynolds number value, $\operatorname{Re}=21,000$, while the lowest one is for the lowest Reynolds number value, $\operatorname{Re}=12,000$. In addition, the use of deflectors with $\operatorname{Re}=21,000$ gives higher friction loss than that with $\operatorname{Re}=12,000,15,000$, and 18,000 around $57.386,41.720$, and $23.157 \%$, respectively. This same figure also show that the use of these same baffle plates yields skin friction rate of about 10.829-25.412 times over that of smooth air channel without baffle plates, depending on the Reynolds number values. 


\section{Heat Transfer Behavior}

The baffle plates have their transverse arrangement of sudden enlargement, which promotes the vortex vertical axes relative to the plane of the fluid flow formed with the bottom wall. The heat transfer coefficient by its global aspect does not distinguish areas where the heat exchange is poor, it was considered useful to conduct a local study of the fluid and then determine the local Nusselt number in the channel. Figure 13 (a) show the normalized evolution of the local Nusselt number $\left(\mathrm{Nu}_{\mathrm{x}} / \mathrm{Nu}_{0}\right)$ calculated along the heated upper channel wall. It is obvious that the minimum value of heat transfer rate is at the base of these plates while it takes its maximum value on its upper sides. This same figure shows that in the areas downstream of the deflectors, the $\mathrm{Nu}_{\mathrm{x}}$ is enhanced. This enhancement is due to the intense mixing by the recirculation flow. As discussed by Nasiruddin and Kamran Siddiqui [5], a recirculation induces a rotary motion inside the flow field, which enhances mixing by brings the parcels of fluid from the near-wall region to the bulk and vice versa. Therefore, the vortices with longer streamwise extent and higher vorticity will contribute more to the mixing and thus, the heat transfer. The heat transfer rate for air flowing in the flat rectangular baffled channel with larger Reynolds number value is found to be higher than that with smaller $\mathrm{Re}$ value. $\mathrm{The} \mathrm{Nu}_{\mathrm{x}} / \mathrm{Nu}_{0}$ rate increases with the increase of Reynolds number and thus, the $\mathrm{Re}=$ 21,000 provides maximum heat transfer.

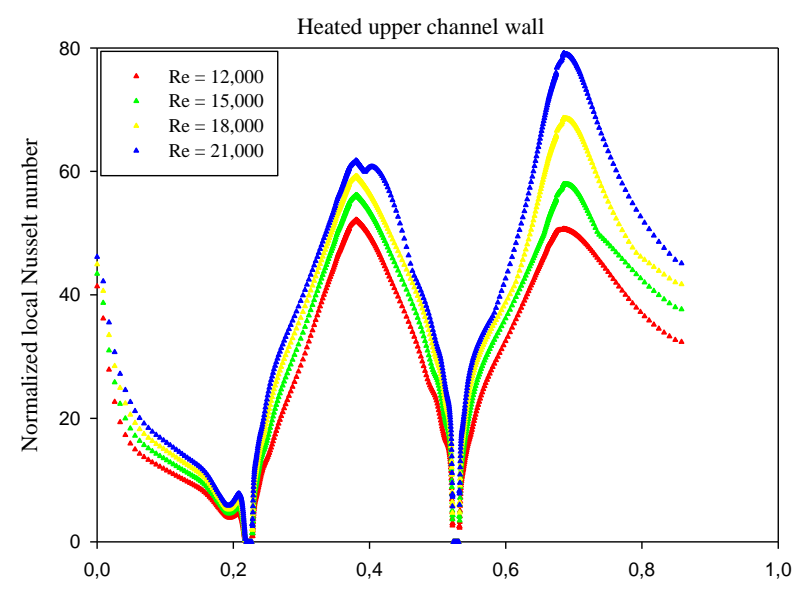

a)

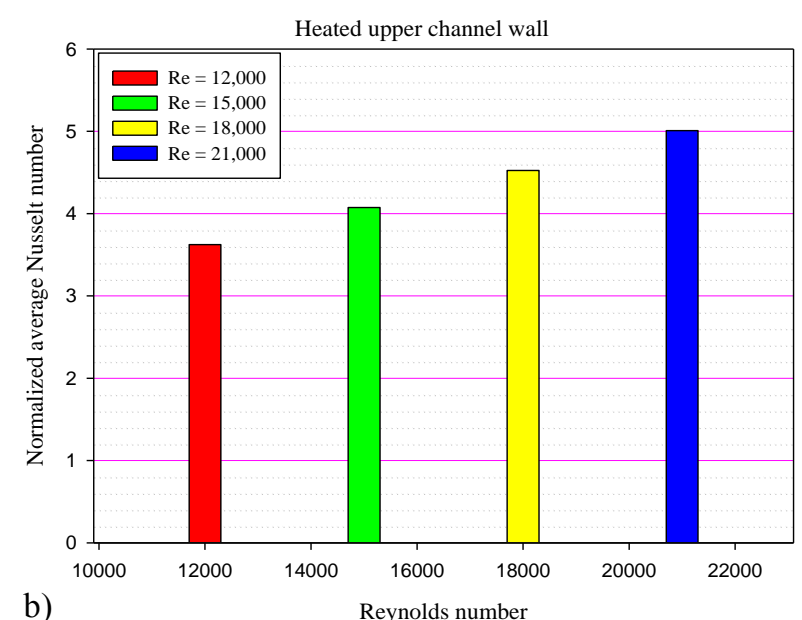

b)

Reynolds number

Figure 13. Variations of normalized a) local and b) average Nusselt numbers with Reynolds numbers

In thermal point of view, it is interesting to study the impact of the variation of the Re value on the evolution of the average Nusselt number along the heated top wall of the channel. Figure 13 (b) shows this dependence. For a Reynolds number realized between 12,000 and 21,000, it was found that the highest rate of heat transfer is achieved by increasing the Re number where the flow structure is very disturbed which promotes mixing of the fluid. On this same wall, when the Reynolds number increases, the Nusselt number believed, where we also find that the temperature gradient at the level of the heated walls decreases with increasing flow rate. This is because the introduction of the negative velocity of the turbulent flow, forced convection reduces the level of turbulence intensity within the boundary layer. The average increase in the Nusselt number of using the baffle plates is in a range of 3.623 to 5.008 times over the smooth air channel with no deflectors. Importantly, the flat plates with $\mathrm{Re}=21,000$ show higher $\mathrm{Nu} / \mathrm{Nu}_{0}$ than the one with $\mathrm{Re}=12,000,15,000$, and 18,000 around 27.664, 18.657 , and $9.657 \%$, respectively.

\section{Thermo-aerodynamic Performance}

Figure 14 shows the variation of the thermal enhancement factor at various Reynolds number values. In this same figure, the thermal performance enhancement factor (TEF) tends to increase with the rise of Reynolds number. For $\mathrm{Re}=12,000,15,000,18,000$, and 21,000, the highest TEF values are 1.651, 1.674, 1.697, and 1.783, respectively. Consequently, the TEFs are seen to be above the unity for all Reynolds numbers and vary between 1.651 and 1.783, depending on the Re values. What was also remarked, the use of flat deflectors with $\operatorname{Re}=21,000$ gives higher $\mathrm{TEF}$ than that with $\mathrm{Re}=12,000,15,000$, and 18,000 around 7.388, 6.086, and 4.182\%, respectively. Therefore, the $\operatorname{Re}=21,000$ may be selected as the best functioning regime to enhance efficiently the heat transfer phenomenon in a channel containing flat rectangular baffle plates. 


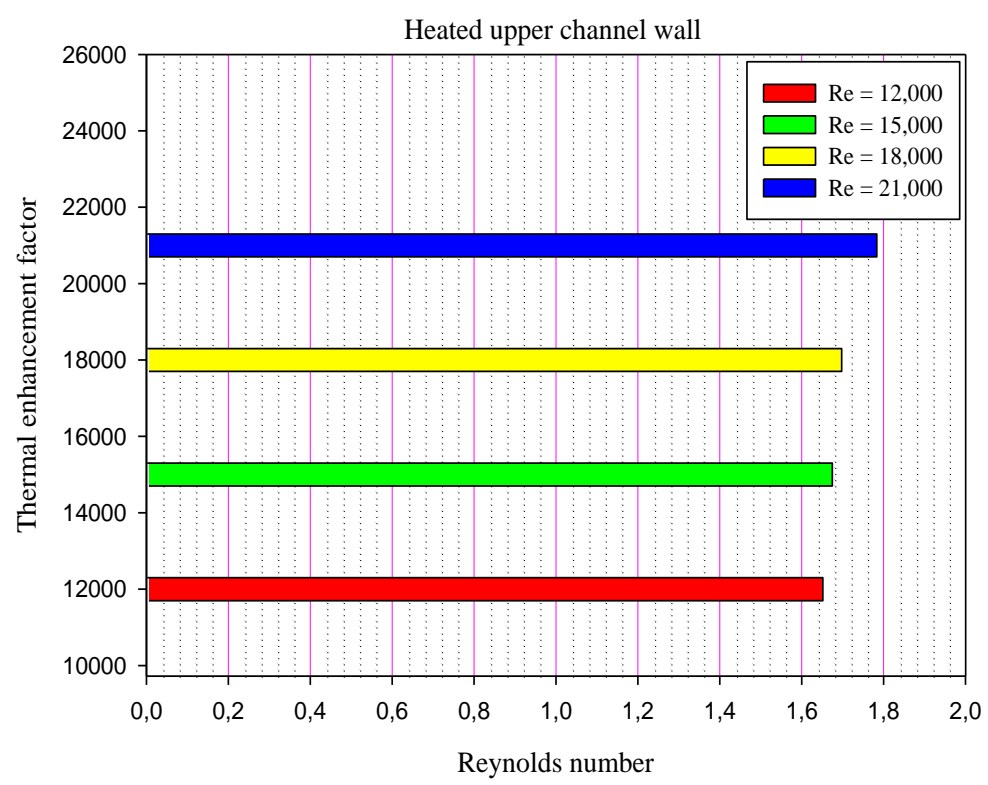

Figure 14. Variation of thermal enhancement factor with Reynolds number

\section{CONCLUDING REMARKS}

Thermo-aeraulic performance of a constant property Newtonian fluid flow through a two-dimensional rectangular channel fitted with four transverse staggered flat rectangular baffle plate deflectors is obtained using the numerical finite volume method. The characterization of the flow and temperature fields in this channel intend to allow the comprehension of the behavior of dynamic loads, produced by the fluctuating velocity and turbulence fields, and contributes to the characterization of heat transfer distribution in heat exchangers. The order of heat transfer improvement is about $362.302-500.866 \%$ for using the flat baffles. However, as expected, the increase is associated with enlarged pressure loss ranging from 10.829 to 25.412 times above the smooth air channel with no plates and in general, high Reynolds number values lead to the best functioning regime in a channel containing baffle plates.

\section{Acknowledgments}

This research was funded by the Unit of Research on Materials and Renewable Energies, Abou Bekr Belkaid University, Republic of Algeria. The authors would like to thank Prof. Dr. Ahmed AZZI for suggestions.

\section{NOMENCLATURE}

Cf Skin friction coefficient

$\mathrm{Cp} \quad$ Pressure coefficient

$\mathrm{C}_{1 \varepsilon} \quad$ Constant used in the standard $\mathrm{k}-\varepsilon$ model

$\mathrm{C}_{2 \varepsilon} \quad$ Constant used in the standard k- $\varepsilon$ model

$\mathrm{C}_{\mu} \quad$ Constant used in the standard k- $\varepsilon$ model

$\mathrm{D}_{\mathrm{h}} \quad$ Aeraulic diameter of the rectangular channel

f Friction factor

$\mathrm{f}_{0} \quad$ Friction factor

$\mathrm{G}_{\mathrm{k}} \quad$ Turbulent kinetic energy production rate

$\mathrm{H} \quad$ Height of air tunnel in channel

h Baffle height

$\mathrm{h}_{\mathrm{x}} \quad$ Local convective heat transfer coefficient

k Turbulent kinetic energy

L Channel length

$\mathrm{L}_{\text {in }} \quad$ Inlet distance

Lout Outlet distance

$\mathrm{Nu} \quad$ Average Nusselt number

$\mathrm{Nu}_{\mathrm{x}} \quad$ Local Nusselt number

$\mathrm{Nu}_{0} \quad$ Dittus-Boelter correlation for smooth channel 
P $\quad$ Fluid pressure

$\mathrm{P}_{\mathrm{atm}} \quad$ Atmospheric pressure

$\mathrm{Pi} \quad$ Separation distance between obstacles

Pr Prandtl number

T Temperature

$\mathrm{T}_{\text {in }} \quad$ Inlet temperature

$\mathrm{T}_{\mathrm{w}} \quad$ Wall temperature

$\mathrm{u} \quad$ Fluid velocity in the $\mathrm{x}$-direction

$\mathrm{u}_{\mathrm{i}} \quad$ Fluid velocity in the $\mathrm{x}_{\mathrm{i}}$-direction

$\mathrm{U}_{\text {in }} \quad$ Inlet velocity

$\mathrm{U}_{\max } \quad$ Maximum axial velocity

$\bar{U} \quad$ Channel average velocity

$\mathrm{v} \quad$ Fluid velocity in the y-direction

w Baffle thickness

W Channel width

$\mathrm{x}, \mathrm{y} \quad$ Cartesian coordinates

$\varepsilon \quad$ Turbulent dissipation rate

$\Gamma \quad$ Molecular thermal diffusivity

$\Gamma_{\mathrm{t}} \quad$ Turbulent thermal diffusivity

$\mathrm{K}_{\mathrm{f}} \quad$ Thermal conductivity of fluid

$\mu \quad$ Dynamic viscosity

$\mu_{\mathrm{t}} \quad$ Eddy viscosity

$v \quad$ Kinematic viscosity

$\rho \quad$ Fluid density

$\sigma_{\mathrm{k}} \quad$ Constant used in the standard $\mathrm{k}-\varepsilon$ model

$\sigma_{\varepsilon} \quad$ Constant used in the standard $\mathrm{k}-\varepsilon$ model

$\tau_{\mathrm{w}} \quad$ Wall shear stress

$\phi \quad$ Stands for the dependent variables $\mathrm{u}, \mathrm{v}, \mathrm{T}, \mathrm{k}$ and $\varepsilon$

$\Delta \mathrm{P} \quad$ Pressure drop

$\sigma_{\mathrm{k}} \quad$ Constant used in the standard $\mathrm{k}-\varepsilon$ model

atm Atmosphere

f Fluid

in Inlet

max Maximum

out Outlet

$\mathrm{t} \quad$ Turbulent

w Wall

$\mathrm{x} \quad$ Local

\section{REFERENCES}

[1] Patankar, S. V., Liu, C. H., Sparrow, E. M. (1977). Fully developed flow and heat transfer in ducts having streamwise-periodic variations of cross-sectional area. Journal of Heat Transfer, 99(2), 180-186.

[2] Guo, Z., Anand, N. K. (1997). Three-dimensional heat transfer in a channel with a baffle in the entrance region. Numerical Heat Transfer, Part A Applications, 31(1), 21-35.

[3] Demartini, L. C., Vielmo, H. A., Möller, S. V. (2004). Numeric and experimental analysis of the turbulent flow through a channel with baffle plates. Journal of the Brazilian Society of Mechanical Sciences and Engineering, 26(2), 153-159.

[4] Gajusingh, S. T., Shaikh, N., Siddiqui, K. (2010). Influence of a rectangular baffle on the downstream flow structure. Experimental Thermal and Fluid Science, 34(5), 590-602.

[5] Siddiqui, M. K. (2007). Heat transfer augmentation in a heat exchanger tube using a baffle. International Journal of Heat and Fluid Flow, 28(2), 318-328.

[6] Karwa, R., Maheshwari, B. K., Karwa, N. (2005). Experimental study of heat transfer enhancement in an asymmetrically heated rectangular duct with perforated baffles. International Communications in Heat and Mass Transfer, 32(1-2), 275-284.

[7] Guerroudj, N., Kahalerras, H. (2010). Mixed convection in a channel provided with heated porous blocks of various shapes. Energy conversion and management, 51(3), 505-517.

[8] Santos, N. B., de Lemos, M. J. (2006). Flow and heat transfer in a parallel-plate channel with porous and solid baffles. Numerical Heat Transfer, Part A: Applications, 49(5), 471-494. 
[9] Kumar, A., Kim, M. H. (2015). Convective heat transfer enhancement in solar air channels. Applied Thermal Engineering, 89, 239-261.

[10] Sripattanapipat, S., Promvonge, P. (2009). Numerical analysis of laminar heat transfer in a channel with diamond-shaped baffles. International Communications in Heat and Mass Transfer, 36(1), 32-38.

[11] Wang, F., Zhang, J., Wang, S. (2012). Investigation on flow and heat transfer characteristics in rectangular channel with drop-shaped pin fins. Propulsion and power research, 1(1), 64-70.

[12] Zhang, J. F., He, Y. L., Tao, W. Q. (2009). 3D numerical simulation on shell-and-tube heat exchangers with middle-overlapped helical baffles and continuous baffles-Part I: Numerical model and results of whole heat exchanger with middle-overlapped helical baffles. International Journal of Heat and Mass Transfer, 52(23-24), 5371-5380.

[13] Won, S. Y., Burgess, N. K., Peddicord, S., Ligrani, P. M. (2004). Spatially resolved surface heat transfer for parallel rib turbulators with $45 \mathrm{deg}$ orientations including test surface conduction analysis. Journal of heat transfer, 126(2), 193-201.

[14] Thianpong, C., Yongsiri, K., Nanan, K., Eiamsa-Ard, S. (2012). Thermal performance evaluation of heat exchangers fitted with twisted-ring turbulators. International Communications in Heat and Mass Transfer, 39(6), 861-868.

[15] Bekele, A., Mishra, M., Dutta, S. (2011). Effects of delta-shaped obstacles on the thermal performance of solar air heater. Advances in Mechanical Engineering, 3, 103502.

[16] Zhou, G., Ye, Q. (2012). Experimental investigations of thermal and flow characteristics of curved trapezoidal winglet type vortex generators. Applied Thermal Engineering, 37, 241-248.

[17] Saini, S. K., Saini, R. P. (2008). Development of correlations for Nusselt number and friction factor for solar air heater with roughened duct having arc-shaped wire as artificial roughness. Solar Energy, 82(12), 1118-1130.

[18] Menni, Y., Azzi, A., Zidani, C., Benyoucef, B. (2016). Numerical analysis of turbulent forced-convection flow in a channel with staggered 1-shaped baffles. Journal of New Technology and Materials, 6(2), 44-55.

[19] Bopche, S. B., Tandale, M. S. (2009). Experimental investigations on heat transfer and frictional characteristics of a turbulator roughened solar air heater duct. International Journal of Heat and Mass Transfer, 52(11-12), 2834-2848.

[20] Jedsadaratanachai, W., Jayranaiwachira, N., Promvonge, P. (2015). 3D numerical study on flow structure and heat transfer in a circular tube with V-baffles. Chinese Journal of Chemical Engineering, 23(2), 342-349.

[21] Promvonge, P. (2010). Heat transfer and pressure drop in a channel with multiple $60 \mathrm{~V}$-baffles. International Communications in Heat and Mass Transfer, 37(7), 835-840.

[22] Chamoli, S., Thakur, N. S. (2016). Correlations for solar air heater duct with V-shaped perforated baffles as roughness elements on absorber plate. International Journal of Sustainable Energy, 35(1), 1-20.

[23] Jedsadaratanachai, W., Boonloi, A. (2014). Effects of blockage ratio and pitch ratio on thermal performance in a square channel with 30 double V-baffles. Case Studies in Thermal Engineering, 4, 118-128.

[24] Kumar, A., Bhagoria, J. L., Sarviya, R. M. (2008). Heat transfer enhancement in channel of solar air collector by using discrete w-shaped artificial roughened absorber. In 19th National 8th ISHMT-ASME heat and mass transfer conference.

[25] Sriromreun, P., Thianpong, C., Promvonge, P. (2012). Experimental and numerical study on heat transfer enhancement in a channel with Z-shaped baffles. International Communications in Heat and Mass Transfer, 39(7), 945-952.

[26] B.E. Launder, D.B. Spalding (1974). The numerical computation of turbulent flows. Computer Methods in Applied Mechanics and Engineering 3, 269-289.

[27] F. Incropera, P.D. Dewitt (1996). Introduction to heat transfer. 3rd edition John Wiley Sons Inc.

[28] Patankar, S. (1980). Numerical heat transfer and fluid flow. CRC press.

[29] Leonard, B. P., Mokhtari, S. (1990). ULTRA-SHARP nonoscillatory convection schemes for high-speed steady multidimensional flow.

[30] Van Doormaal, J. P., Raithby, G. D. (1984). Enhancements of the SIMPLE method for predicting incompressible fluid flows. Numerical heat transfer, 7(2), 147-163.

[31] Fluent, I. N. C. (2006). FLUENT 6.3 user's guide. Fluent documentation.

[32] Pirouz, M. M., Farhadi, M., Sedighi, K., Nemati, H., Fattahi, E. (2011). Lattice Boltzmann simulation of conjugate heat transfer in a rectangular channel with wall-mounted obstacles. Scientia Iranica, 18(2), 213-221.

[33] Lin, C. W. (2006). Experimental study of thermal behaviors in a rectangular channel with baffle of pores. International communications in heat and mass transfer, 33(8), 985-992. 